\title{
The Origin of Conductive-Pulse Sensing Inside a Nanopore and the Role of Electro-Hydrodynamics
}

\author{
Lauren S. Lastra ${ }^{1}$, Michelle Nguyen ${ }^{2}$, Nasim Farajpour ${ }^{1}$, Kevin J. Freedman ${ }^{1 *}$ \\ ${ }^{1}$ Department of Bioengineering, University of California, Riverside, 900 University Ave., \\ Riverside, CA 92521, USA \\ ${ }^{2}$ Department of Biology, University of California, Riverside, 900 University Ave., Riverside, CA \\ 92521, USA \\ *Correspondence to: Kevin J. Freedman, Department of Bioengineering, University of \\ California, Riverside, 900 University Ave., Riverside, CA 92521, USA. E-mail: \\ kfreedman@engr.ucr.edu
}

\begin{abstract}
Despite the highly negatively charged backbone of DNA, electroosmotic flow (EOF) within a nanopore can lead to DNA travelling opposite to electrophoretic forces (EPF) at low ionic strengths. However, EOF pumping and its role in producing current-enhancing events is ambiguous due to the complicated interactions between nanopore walls, DNA grooves, ion mobility, and counterion clouds. Here, we discuss how current-enhancing DNA events could be the result of a flux imbalance between anions and cations. The contributing factors for driving a flux imbalance within a nanopore include pore size, voltage bias, and type of alkali chloride electrolyte. Once the mechanism behind conductive events is established, the physics of transducing a DNA translocation into an electrical signal can be further exploited for improving DNA sequencing and, more broadly, bio-sensing.
\end{abstract}

\section{Introduction}

Since their first use as a biosensor, solid-state nanopores continue to explore new biophysical phenomena and have cemented their place in history as invaluable real-time, singlemolecule, electrical read-out platforms. Although the translocation of new biological entities is now a routine practice in labs across the world, the high electrolyte concentrations in which experiments are performed is rather unchanged since nanopores were first utilized in $1996^{1}$. The popularity associated with high electrolyte solution is largely due to the high signal-to-noise ratio (SNR) and reliable generation of resistive pulses stemming from DNA transiently blocking ions (typically potassium and chloride). The physical principles in which DNA modulates the flow of ionic current within a nanopore has been studied extensively ${ }^{2-4}$. However, the resistive nature of events is not consistent across all DNA translocation experiments ${ }^{1,5,6}$. In 2004, Chang et. al., reported on current-enhancing events wherein the DNA-occupied pore conducts more ions than when the pore is empty ${ }^{7}$. Therefore, events can be categorized as a current-reducing event (i.e. resistive event, RE), or a current-enhancing event (i.e. conductive event, $\mathrm{CE}$ ). The question, "Why does ionic current increase during transient DNA occupancy of a nanopore?", remains unanswered and warrants further investigation.

As electrolyte concentration decreases, CEs are often observed in both planar membrane nanopores as well as conical nanopipettes, suggesting that CEs are not pore geometry specific ${ }^{8-16}$. It is also at this regime where EOF strengthens, sometimes leading to the translocation of molecules opposite of EPF (i.e. negative DNA traveling towards the negatively biased electrode). 
Although EOF and CEs often coincide, it is important to note that they are not mechanistically linked. Reports of CEs occurring in nanopores where EOF is reduced to allow EPF-driven events also produced $\mathrm{CEs}^{9}$. Despite the large number of experiments describing CEs, the origins of CEs in the presence of low ionic strength has been elusive.

It has been generally accepted that CEs stemming from low ionic strength conditions occur because the introduction of additional DNA counterions (i.e. $\mathrm{K}^{+}$) within the nanopore is greater than the number of ions within the empty pore ${ }^{7}$. Once electrolyte concentration decreases below roughly $0.02 \mathrm{M}$, mostly counterions are present within the pore, which explains the current enhancement $^{17,18}$. Interestingly, at approximately $0.4 \mathrm{M}$, counterions are thought to precisely compensate for the DNA occupied regions of the pore and yields no current modulation ${ }^{19}$. A second hypothesis has been that frictional forces (i.e. ionic friction with the grooves on DNA) are influential in generating $\mathrm{CEs}^{3,9}$.

Although these hypotheses can predict the well-known crossover point in which events transition from resistive to conductive (via decreasing salt concentrations), the cation-specific, voltage-specific, and pore size-specific dependence of CEs have not been studied and should provide confirming evidence for one of these hypotheses ${ }^{6,19}$. One recent experiment in particular conflicts with these hypotheses and may lead to a third potential theory; namely that current enhancement is not only a low salt phenomena but can also be observed at high, asymmetric salt conditions $^{20}$. Above $1 \mathrm{M} \mathrm{KCl}$, counterions should contribute very little to current modulation, which motivated us to explore event properties within this regime. Since a cohesive theory for the nature of conducting events is still elusive, we studied the transport of DNA within a nanopore using various monovalent salts.

Herein, we characterize EOF-driven events (anti-electrophoretic, or anti-EPF) with Lambda DNA ( $\lambda$-DNA) and neutral polymers (i.e. polyethylene glycol, PEG) using quartz nanopores. Interestingly, we found that current enhancements can be observed using PEG; casting doubt on counterions being the dominant explanation for CEs. Furthermore, DNA CEs are extremely cation-, pore size-, and voltage-specific and may be the result of an imbalance of ionic fluxes. We will discuss the electrokinetic and hydrodynamic phenomena that affect event shape such as counterion cloud, ion mobility, pore size, and electrolyte composition. This report elucidates some of the fundamental prerequisites for observing CEs when DNA translocates a nanopore and paves the way for harnessing CE mechanisms for DNA sequencing and biophysical discoveries.

\section{Results}

While most nanopore-based, single-molecule sensing is performed using planar membranes, which have a well-defined pore length (i.e. membrane thickness), nanopipettes have a gradual taper length (Fig. 1a) that increases the sensing region of the device ${ }^{21}$. We fabricate nanopipettes by laser pulling glass nanocapillaries, producing two identical quartz nanopores. With this technique, we can achieve $<10 \mathrm{~nm}$ inner pore diameters, Figure 1a. This process is fast, inexpensive, and does not require a clean-room environment ${ }^{22}$.

Current-voltage (I/V) analysis reveals that the conductance of the pores (Fig. 1b) varies between 0.58 and $5.35 \mathrm{nS}$; as well as the presence of ionic current rectification ${ }^{23}$. These conductance values are consistent with pore diameters between $5( \pm 0.5)$ and $48( \pm 4) \mathrm{nm}$, 
respectively. Specifically, the relationship between pore conductance $(G)$ and inner diameter $\left(d_{i}\right)$ allows us to estimate the size of the aperture ${ }^{24,25}$ :

$$
d_{i}=\frac{4 G l}{\pi g d_{b}}
$$

Where $l$ is the length of the conical pore (taper length), $g$ is the measured conductivity of the buffer, and $d_{b}$ is the diameter of the capillary at the beginning of the conical taper. The initial, inner capillary diameter is constant in our experiments $(0.7 \mathrm{~mm})$ and the buffer conductance depends on concentration and alkali chloride used. The taper length was measured using transmission electron microscopy and I/V analysis is used to measure the pore conductance. Pore sizes are also occasionally confirmed using transmission electron microscopy.

After retrieving I/V information, translocation experiments with $\lambda$-DNA at $500 \mathrm{pM}$ were performed in $10 \mathrm{mM}$ Tris-EDTA (pH 7.4). When EPF dominates, the capture volume outside the nanopore assumes a nearly spherical shape surrounding the pore's oriface ${ }^{26-30}$. As ionic strength decreases, EOF can dominate as the primary means for DNA entering the pore but is $\mathrm{pH}$ dependent since a high surface charge on the glass is required ${ }^{31}$. According to the EOF streamlines, the capture volume adopts a shape confined along the sides of the pore ${ }^{32}$. There also lies a crossover concentration point in which EOF reverses direction, where EOF is generated along the glass surface and radiates away from the pore aperature ${ }^{32}$.

Finite element analysis was performed to determine the fluid flow rate at different voltages (Fig. 1c). As the applied voltage decreases from $0 \mathrm{mV}$, the mean fluid velocity increases into the glass pore. The same is true for positive voltages, however, the fluid flow direction switches (flow reversal) from towards the pore at negative voltages to away from the pore at positive voltages. Notably, these fluid velocities can influence DNA dwell time inside the pore and have been described using hydrodynamic drag ${ }^{33,34}$.

DNA proceeds to diffuse around the solution until it enters the EOF capture volume, where it is then transported through the pore. This method of translocation is further illustrated (Fig. 1d) with $\mathrm{KCl}$ as the electrolyte and $\mathrm{K}^{+}$ions responsible for the movement of water carrying $\lambda$-DNA. Under these conditions, it is possible to see differing DNA configurations: linear, partially folded, and fully folded (Fig. 1d). Reports of different DNA configurations have been witnessed using high ionic strength conditions and with both planar nanopores ${ }^{35-38}$ and nanocapillaries ${ }^{24}$. The ability to discriminate folding states using DNA CEs does not directly help uncover the nature of CEs, but it is important to recognize the existence and understand the effects of having various DNA configurations upon translocation.

To show that this finding is not limited to low ionic strength phenomenon, we employed salt concentration gradients as previously described ${ }^{20}$. As shown in Figure 1e, our experimental set-up involved having a solution of $1 \mathrm{M} \mathrm{KCl}+\lambda$-DNA inside the nanopore with $4 \mathrm{M} \mathrm{KCl}$ outside. With an applied voltage of $-600 \mathrm{mV}, \lambda$-DNA was driven outside the pore through EPF, resulting in CEs. An additional salt gradient was implemented (Fig. 1f) with $4 \mathrm{M} \mathrm{KCl}$ inside the nanopore and $1 \mathrm{M} \mathrm{KCl}+\lambda$-DNA outside. In this situation, EPF drove $\lambda$-DNA to translocate through the pore, again resulting in CEs. Based on these results, a working hypothesis was made that the existence of CEs stem from a flux imbalance between anions and cations. This is notably different than ion selectivity which is typically a characteristic of the pore itself. Rather, flux imbalances can be generated through externally applied conditions and parameters. EOF pumping of water 
into the pore, for example, can change the relative fluxes of ions. Since the electric field acts equally on both chloride and potassium ions, the net movement of water only provides a moving frame of reference which favors one ion over another. Nevertheless, total ionic current is constant regardless of EOF velocity. For the data shown where CEs are observed (Fig. $1 \mathrm{~d}$-f) we speculate that there is a net flux that favors potassium ions. Figure $1 \mathrm{~g}$ illustrates how $\mathrm{K}^{+}$ions are pumped into the pore under low ionic strength (EOF; Fig. 1d) and concentration gradient (asymmetric) conditions (EPF; Fig. 1e).

a)

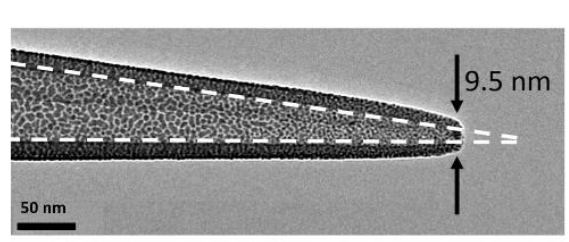

b)

c)
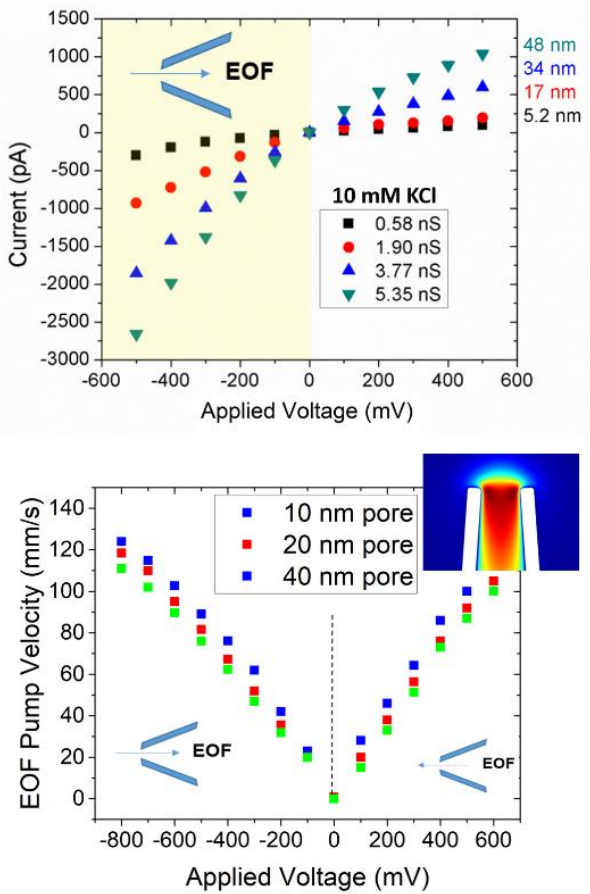

d)

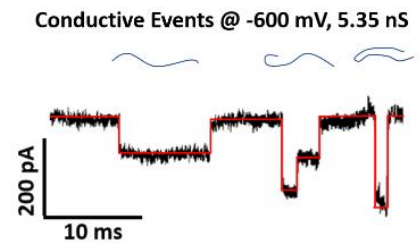

e)

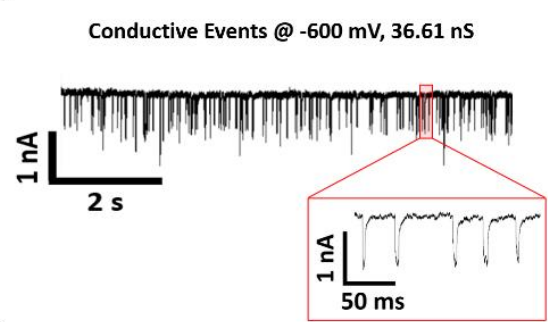

f)

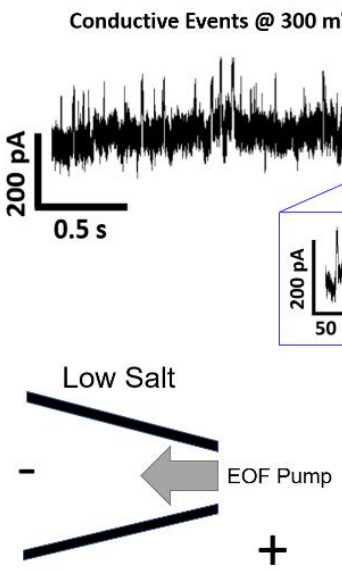

g)

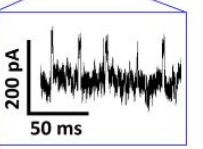

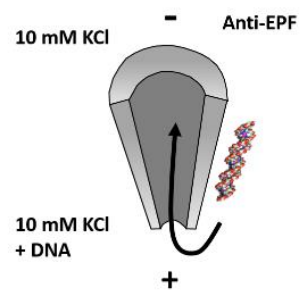

EPF

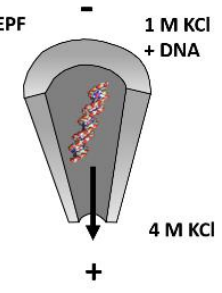

EPF

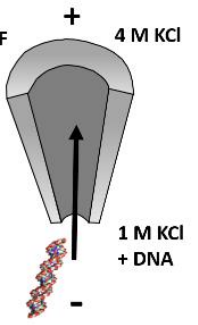

$\mathrm{M} \mathrm{KCl}$

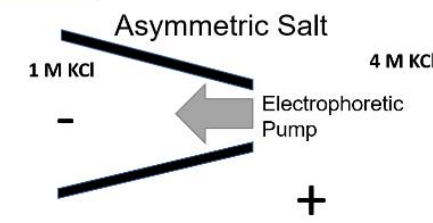

Figure 1: Experimental set-up and characterization of quartz nanopores. (a) TEM of quartz nanopore; scale bar, $50 \mathrm{~nm}$. (b) I/V curves for quartz nanopores for four differently sized pores. (c) Simulations of EOF pump velocity at various voltages within quartz nanopores; pore diameter was $20 \mathrm{~nm}$ and was symmetrically filled with $10 \mathrm{mM} \mathrm{KCl}$. EOF reversal also shown. Inset: fluid velocity distribution near the pore. (d) Schematic and corresponding current trace of linear, partially folded, and fully folded DNA configurations for EOF-driven translocations. (e) Current trace of EPF-driven events at $-600 \mathrm{mV}$ under influence of salt gradient. Red inset: close-up of above current trace to show event signature and SNR. (f) Current trace of EPF-driven events with salt gradient at $300 \mathrm{mV}$. Blue inset: Magnified region of above current trace to show event signature and SNR. (g) Low ionic strength, EOF pumping yields high current-enhancing events as well as electrophoretic pumping of cations from the $4 \mathrm{M} \mathrm{KCl}$ solution into the nanopore. 
Validation using finite element methods was undertaken to further explain the potential impact that EOF has on DNA sensing and the unique capture dynamics of EOF-driven events. A $20 \mathrm{~nm}$ pore was modeled with $10 \mathrm{mM}$ and $2 \mathrm{M}$ salt to demonstrate the difference in capture kinetics of DNA. At low ionic strength conditions, fluid velocity is plotted with colored streamlines indicating where the fluid entering the pore is coming from within the bath solution. Since DNA events only occur anti-EPF, mapping the fluid motion is indicative of the capture zone. To experimentally validate the finite element analysis (Fig. 2a), $\lambda$-DNA was tagged with YOYO-1 and the nanopipette tip placed in the focal plane of a water immersion objective (Nikon, NA=1.2). A stacked timeseries of images (acquired from a Princeton Instruments ProEM EMCCD) allowed us to observe $\lambda$-DNA capture at $-700 \mathrm{mV}$ (Fig. 2a inset reveals that fluid motion along the sides of the pore is responsible for $\lambda$-DNA translocation). At high ionic strength conditions, DNA travels through the pore via EPF and so the electric field lines are plotted (Fig. 2b) and represent the capture zone.

Realizing that the capture volume in EOF-driven translocations surrounds the outer walls of the nanopipette, we chose to expand and shrink the capture volume via a depth-dependent study to witness any changes in event frequency (Fig. 2c). By submerging varying lengths of the taper length inside the salt solution containing $\lambda$-DNA, the capture volume is controlled (Supplementary Fig. 1). The nanopore was suspended at $0,0.26,0.53,1.1$, and $4.0 \mathrm{~mm}$ below the electrolyte solution surface containing DNA. For exact measurements, the nanopore was suspended from a micrometer. Translocations were obtained for voltages between -100 and $-1000 \mathrm{mV}$, in increments of $100 \mathrm{mV}$. Recording at $-600 \mathrm{mV}$ yielded the most consistent translocations without clogging the pore. Events were recorded at $-600 \mathrm{mV}$ and the I/V relationship yielded a $2.53 \mathrm{nS}$ pore. Capture rate was calculated at each depth. As nanopore depth increases, capture volume also increases, leading to higher event frequency with larger depth values. As more of the nanopore is exposed to the $\lambda$-DNA solution, the capture volume enlarges, leading to an increase in event frequency.

In order to understand how electro-hydrodynamics influences ionic flux, particularly at low salt conditions, three monovalent salts were modelled by altering the cation diffusion coefficient and electrophoretic mobility (Fig. $2 \mathrm{~d}$ and e). Although the pore's total ionic flux was not altered significantly by EOF since $\mathrm{K}^{+}$flux increased and $\mathrm{Cl}^{-}$flux decreased by the same amount, EOF does significantly impact the flux imbalance between cation and anion. This finding was particularly noteworthy since CEs have been observed at high asymmetric salt conditions which would also change a pore's ionic flux imbalance. These results predict that a flux imbalance in favor of $\mathrm{Cl}^{-}$transport leads to REs and a flux imbalance in favor of $\mathrm{K}^{+}$leads to CEs. This is based on the experimental results that the $10 \mathrm{mM} \mathrm{KCl}$ electrolyte always produces CEs. In Figure $2 \mathrm{~d}$, anion-dominant flux only occurs with small pore sizes, $20 \mathrm{~nm}$ and less, and an applied negative voltage between -300 and $-400 \mathrm{mV}$. It is important to note that no events could be recorded at these conditions to find out whether resistive events are observed. For a nanopore suspended in $\mathrm{LiCl}$, we observed more opportunities for the pore to be $\mathrm{Cl}^{-}$selective, which we predict will result in REs upon translocation of $\lambda$-DNA. As the pore increases in size or an increasingly negative voltage is applied, the pore can become cation selective, which we speculate can give rise to CEs.

Likewise, a comparison between symmetric low salt conditions and asymmetric high salt conditions was performed using $20 \mathrm{~nm}$ pores (Fig. 2f). As previously stated, a $20 \mathrm{~nm}$ pore under low ionic strength $\mathrm{KCl}$ conditions has few opportunities to have a flux imbalance in favor of $\mathrm{Cl}^{-}$ 
transport. This can occur at positive applied voltages as well as small negative voltages for $\mathrm{KCl}$ (between 0 and $400 \mathrm{mV}$ ). In order to improve on our experimental design and use conditions that favor cations (or anions), asymmetric salt conditions can be useful and have already proved to yield CEs. This was also modelled, in which $1 \mathrm{M} \mathrm{KCl}$ is inside the pore and $4 \mathrm{M} \mathrm{KCl}$ is outside, and the result was that the pore favored $\mathrm{K}^{+}$transport due to electrophoretic pumping of the cation. 
This computational finding further supports the previous experimental observations of CEs occurring under asymmetric salt conditions as DNA translocates the pore.

Although the ionic diffusion coefficients and electrophoretic mobilities encapsulate basic transport properties, all the while being utilized as variables in the finite element simulations, they neglect the geometric size of the ions and therefore the packing density/strength on oppositely

a)

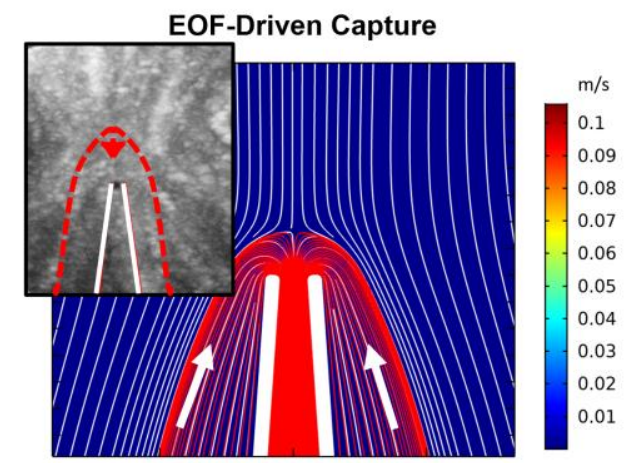

b)
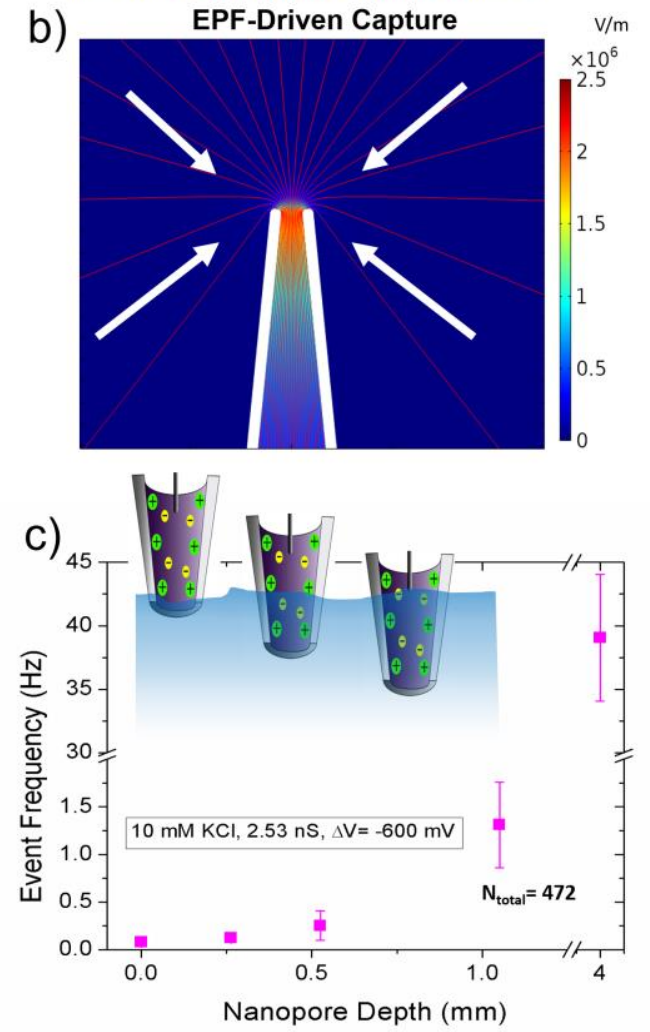

d)

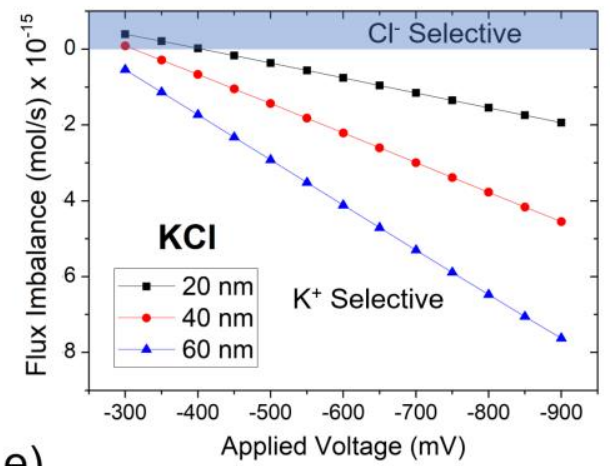

e)

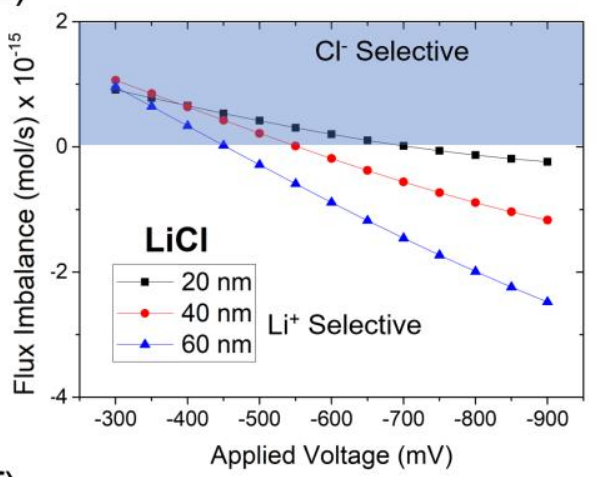

f)

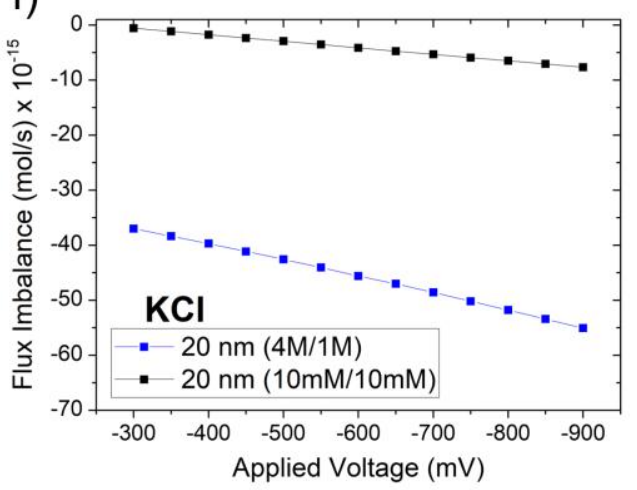

Figure 2: Finite-element modeling and experimental characterization of EOF. Two-dimensional axisymmetric modeling of (a) EOF dominant capture in which white and red lines show fluid streamlines (inset: YOYO-labelled $\lambda$ DNA sample with an applied voltage of $-700 \mathrm{mV}$ ). Red lines depict EOF-capture volume for a $20 \mathrm{~nm}$ pore at a negative $600 \mathrm{mV}$ voltage bias. (b) Electric field lines depicting the expected trajectories of charged molecules during EPF capture. Direction of electric field is shown with white lines at $-600 \mathrm{mV}$. (c) Frequency of events as the pore is increasingly submerged into the $10 \mathrm{mM} \mathrm{KCl}+\lambda$-DNA solution. Error bars made by using the standard deviation of event frequencies. Figures showing ion mobilities of (d) $10 \mathrm{mM} \mathrm{KCl}$ and (e) $10 \mathrm{mM} \mathrm{LiCl}$ for various pore sizes. (f) Flux imbalance comparison between pores (20 nm diameter) with symmetric low salt conditions (10 $\mathrm{mM} / 10 \mathrm{mM})$ and asymmetric high salt conditions (4 M outside/1 $\mathrm{M}$ inside). 
charged surfaces. In order to understand the link between electro-hydrodynamics and Debye layer screening of the quartz surface charge, streaming current measurements were used as a proxy for cation mobility within the diffuse ion layer. Contrary to EOF, where mobile ions drag fluid, streaming currents measure the fluid's ability to drag along ions co-axial to the fluid motion ${ }^{39}$. A pressure bias was used to generate a streaming current and the resulting data can be seen in Supplementary Figure 2. Negative pressures generate a flow into the nanopore and in the same direction as EOF in our experiments. We see that larger pressures create larger streaming currents. Interestingly, $\mathrm{LiCl}$ has significantly higher streaming currents compared $\mathrm{KCl}$ and $\mathrm{CsCl}$ at negatively biased pressures. Overall, these results indicate LiCl-filled nanopores can be $\mathrm{Cl}^{-}$flux dominant at low voltages, and secondly, $\mathrm{Li}^{+}$has a higher flux under pressure biased fluid flow compared to other cations $\left(\mathrm{K}^{+}\right.$and $\left.\mathrm{Cs}^{+}\right)$. The same pore $(1.30 \mathrm{nS}$ in $10 \mathrm{mM} \mathrm{KCl})$ was used for all measurements to reduce variability due to different pore sizes.

\section{DNA and Neutral Polymers in Potassium Chloride}

Under high ionic strength conditions, pores with a diameter slightly larger than the analyte molecule yield greater SNR values when compared to larger pores ${ }^{19}$. Because of this, we were motivated to explore SNR values under low ionic strength conditions. A typical conductive DNA event can be seen in Figure 3a in $10 \mathrm{mM} \mathrm{KCl}$. Potassium chloride was chosen as the electrolyte because it is most frequently utilized in nanopore research due to similar ion mobilities of anions and cations. We incorporated differently sized pore diameters to witness any effect that pore size may have on event shape and size. The depth of each nanopore was kept consistent for all recordings as well as the voltage $(-600 \mathrm{mV})$.

As $\lambda$-DNA translocates through the nanopore, we witness a current-enhancing event. For all SNR calculations, we omitted all configurations except for linear DNA translocations (Fig. 3b). DNA has the ability to translocate linearly, folded ${ }^{8,24}$, or in $\operatorname{knots}^{35}$, in which the latter two increase the current change. To ensure DNA configuration had no effect on SNR, we applied only linearly translocating DNA to our calculation. 
a)
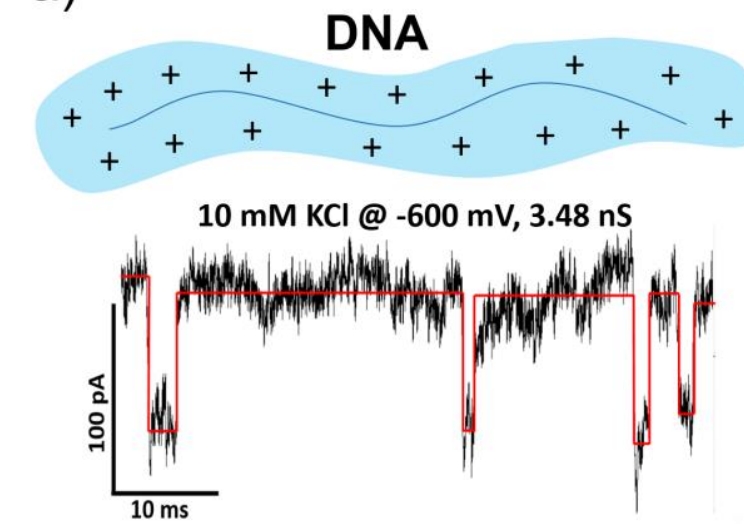

c) PEG

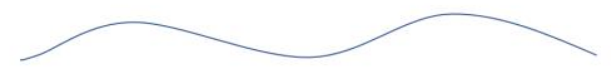

10 mM KCl @ -500 mV, 2.63 nS
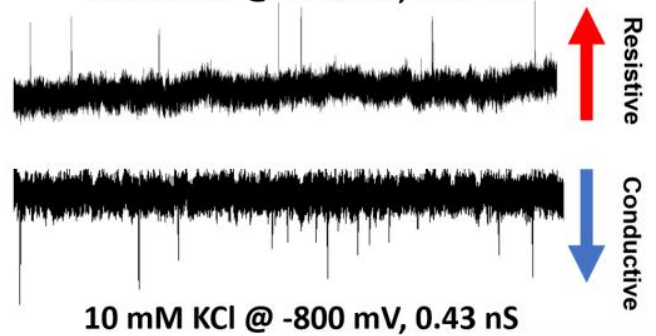

$10 \mathrm{mM} \mathrm{KCl} @-800 \mathrm{mV}, 0.43 \mathrm{nS}$

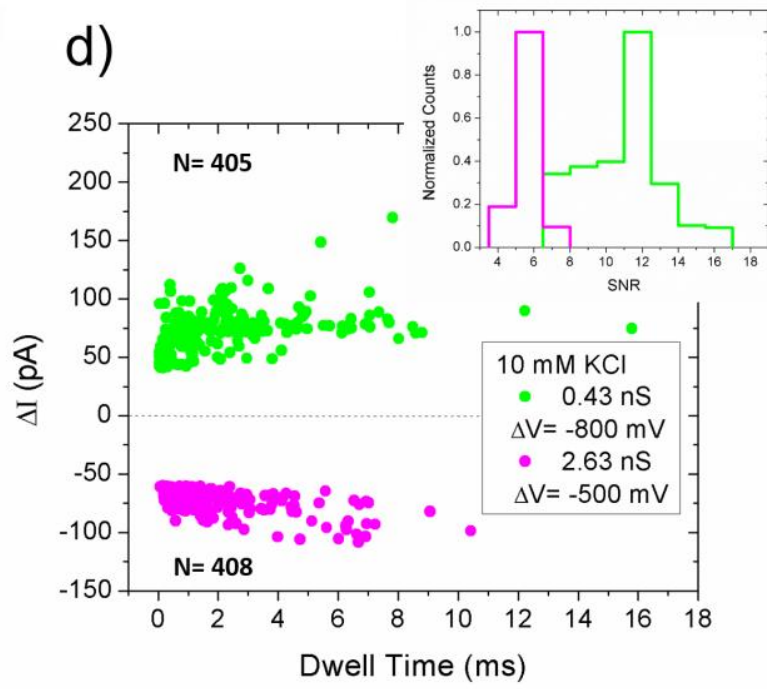

Figure 3: Capture of $\lambda$-DNA and PEG using EOF. (a) Top: depiction of DNA and its counterion cloud. Bottom: current trace of $\lambda$-DNA translocation in $10 \mathrm{mM} \mathrm{KCl}$. (b) SNR calculations for various pore sizes (inset: normalized histogram of data sets from red and blue dotted squares). Each symbol and error bar represent the mean and standard deviation of SNR for one pore. (c) PEG illustration (no counterions present; top) along with current traces of resistive and conductive events (bottom) (d) Scatter plot showing the relationship between current change and dwell time for two different pore sizes (inset: normalized histogram showing SNR between the two aforementioned data sets).

We witness an increase in SNR starting at $2.00 \mathrm{nS}$ and saturating around $3.00 \mathrm{nS}$. To determine whether the current enhancement or the noise of the signal is the major contributor to the increase in SNR, we acquired the median current change of all events and the root mean square (RMS) noise of a data segment lacking events. We witness that the RMS noise maintaining values of $15 \pm 7 \mathrm{pA}$, whereas the current change increases from 30 to $140 \mathrm{pA}$ as pore size increases. For the left side of the graph, we witness a sharp increase in the SNR as the pore size decreases. This can be explained by a decrease in the noise associated with smaller pores. As seen in Supplementary Figure 3, the RMS noise is extremely low ( $<10 \mathrm{pA})$ whereas the median current change is approximately $100 \mathrm{pA}$. Therefore, the higher SNR values for smaller pores stem from lower noise. On the right side of the graph, we speculate that the rise in SNR (and current enhancement) is a result of greater EOF pumping as a function of pore size (Supplementary Fig. 
4). Owing to larger fluid velocities, the flux imbalance highly favors potassium rather than chloride.

The common hypothesis that DNA counterions are the sole mechanism of CEs led us to explore PEG under low ionic strength conditions with an applied negative voltage ${ }^{40}$. PEG 20,000 was diluted to $15 \%$ (w/w) in $10 \mathrm{mM} \mathrm{KCl}$ and voltage was applied from -100 to $-1000 \mathrm{mV}$, in increments of $100 \mathrm{mV}$. PEG events were independently observed with 5 different pores. Interestingly, CEs were most notable at extremely small pore sizes (e.g. $0.43 \mathrm{nS}$ ); a pore size regime that we could not observe DNA events. Since EOF flow rate (e.g. fl/s) decreases with smaller pore sizes and EPF increases, we believe DNA could not energetically overcome the barrier at the pore entrance for translocations to occur. Since PEG is neutral, we were able to observe EOF-driven events at very small pore sizes (Supplementary Fig. 5). The results indicated that smaller pore sizes resulted in CEs whereas larger pore translocations yielded REs (Fig. 3c and d). SNR calculations showed that the smaller pore diameter yielded higher SNR values in comparison to larger pore diameters. In both pores, the median current change was $71 \pm 1 \mathrm{pA}$; whereas the RMS noise increased from 7 to $18 \mathrm{pA}$ as the pore size increased from 4 to $25 \mathrm{~nm}$ in diameter, respectively. Based on these results, the nature of the event (CEs versus REs) seems uncoupled from the analyte counterions (or lack thereof, in the case of PEG) but rather linked to the pore size and/or voltage in which translocations occur. For the same conductance (i.e. nanopore size) of $2.6 \mathrm{nS}$, DNA yielded CEs and PEG yielded REs. Two other possibilities exist for why PEG and DNA produce different event signatures at this pore size: (1) PEG could be interacting with the charged glass surface far from the pore and could impact EOF pumping ${ }^{9}$ and therefore could be impacting the pore's flux imbalance or (2) the size of PEG (150-fold smaller in length compared to $\lambda$-DNA) limits the extent in which EOF pumping is blocked. Nevertheless, it is clear that the analyte counterions do not seem to play a significant role in generating CEs for extremely small, negatively charged pores.

\section{Voltage Dependence with Lithium Chloride}

Lithium chloride was chosen as an electrolyte because it has been previously shown to "slow-down" DNA translocations under high ionic strength conditions ${ }^{41}$. This can be attributed to $\mathrm{Li}^{+}$having a smaller atomic radius than $\mathrm{K}^{+}$and therefore, $\mathrm{Li}^{+}$binds to DNA stronger than $\mathrm{K}^{+41}$. Additionally, $\mathrm{LiCl}$ had a significantly higher streaming current (Supplementary Fig. 2) compared to both $\mathrm{KCl}$ and $\mathrm{CsCl}$. Finite element simulations indicated a voltage and pore size dependence for flux imbalance that was within the voltage range: -400 to $-1000 \mathrm{mV}$, where events are typically observed. The nanopore containing $10 \mathrm{mM} \mathrm{LiCl}$ was inserted inside a solution containing $10 \mathrm{mM}$ $\mathrm{LiCl}+\lambda$-DNA and current changes were recorded at various voltages (Fig. 4a). The same series of steps were repeated to calculate the SNR at each voltage.

Using the same pore $(1.20 \mathrm{nS})$, we witnessed the crossover point that is independent of salt concentration, which is something not previously observed. At voltages of -300 and $-500 \mathrm{mV}, \lambda$ DNA translocations resulted in REs and at voltages of -700 and $-900 \mathrm{mV}$, DNA translocations resulted in CEs, as shown in Figure 4b. Interestingly, at an applied voltage of $-600 \mathrm{mV}$ the event current shape assumes both a resistive and conductive spike (Supplementary Fig. 6). For this pore, we see an increase in the amplitude of the REs as the voltage applied is reduced to $-600 \mathrm{mV}$. Less than $-600 \mathrm{mV}$ (i.e. more negative), the $\mathrm{CE}$ amplitude continues to increase as the voltage decreases 
to $-900 \mathrm{mV}$. The events recorded at $-900 \mathrm{mV}$ and $-500 \mathrm{mV}$ yielded higher $\mathrm{SNR}$ values in comparison to $-700 \mathrm{mV}$ and $-300 \mathrm{mV}$, respectively (Fig. 4c and d). Supplementary Figure 7 shows how the median current change is the main contributor to the SNR fluctuation, the RMS noise for each voltage remains relatively constant. The transition from REs to CEs can be understood by the pore being anion selective at low voltages and cation selective at higher voltages. The respective flux imbalances for each voltage applied can be seen in Supplementary Figure 8. As the applied voltages increase in negativity, the change in current switches to a $\mathrm{CE}$. The biphasic nature of the events at the transitional voltages $(-500 \mathrm{mV}$ and $-700 \mathrm{mV})$ suggests that there may be two mechanisms of current modulation (hydrodynamic flow and pore occupancy) that can occur when the DNA molecule is near or entering the pore. DNA entering the flow field of the pore during EOF pumping may cause current modulations that occur immediately prior to translocation.

Another comparison was done using two pores with inner diameters of $33 \pm 3 \mathrm{~nm}$. One pore contained $10 \mathrm{mM} \mathrm{KCl}$ and was suspended in $10 \mathrm{mM} \mathrm{KCl}+\lambda$-DNA while the other contained $10 \mathrm{mM} \mathrm{LiCl}$ and was suspended in $10 \mathrm{mM} \mathrm{LiCl}+\lambda$-DNA. Both had an applied voltage of -600 $\mathrm{mV}$ and we witnessed $\mathrm{CEs}$ for the pore containing $\mathrm{KCl}$ and $\mathrm{REs}$ for $\mathrm{LiCl}$ (Fig. 4e). At $-600 \mathrm{mV}$ with the aforementioned pore size, finite element simulations predicted that the nanopipette is cation selective in $\mathrm{KCl}$ and anion selective in $\mathrm{LiCl}$, which may be a possible explanation for the event types observed. We also note that $\mathrm{KCl}$ and $\mathrm{LiCl}$ have similar event durations at these low salt conditions, however, $\mathrm{KCl}$ has a much larger variation in the degree of current-modulation (in the case of $\mathrm{KCl}$ : current enhancement). The current-reductions observed for $\mathrm{LiCl}$ are much more tightly clustered together compared to $\mathrm{KCl} \mathrm{CEs}$. The source of the variability observed in $\mathrm{KCl} \mathrm{CEs}$ is still not fully understood and requires further investigation. The data seems to suggest that CEs are more variable regardless of the cation. The $\mathrm{LiCl}$ events in Fig. 4b, for example, show a much greater degree of scatter for CEs compared to REs. 


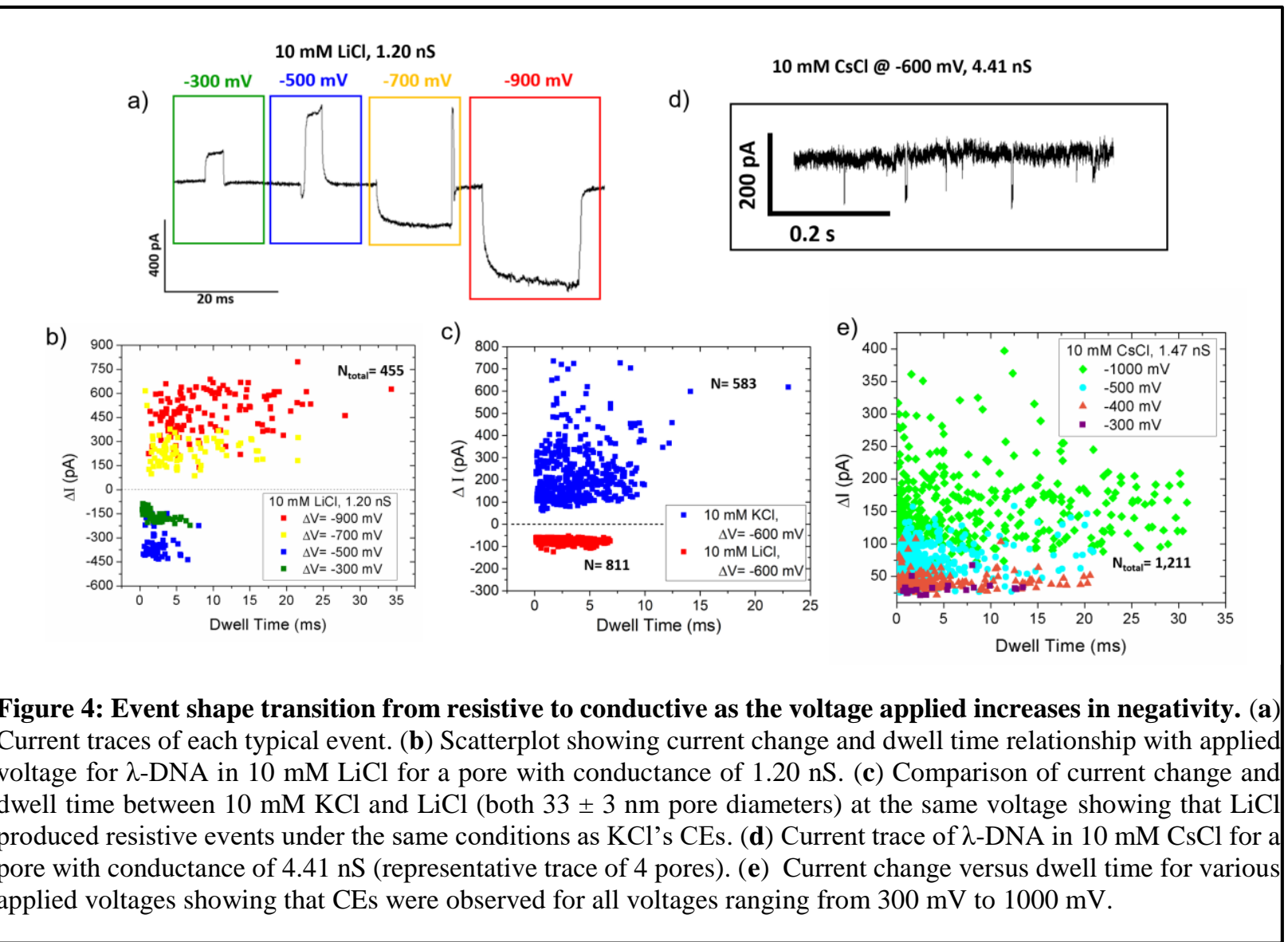

\section{Alkali Chloride Dependence on Event Characteristics}

Recently, $\mathrm{CsCl}$ was shown to have an advantage over $\mathrm{KCl}$ in respect to sequencing using solid-state nanopores ${ }^{11}$. This publication used $\mathrm{CsCl}$ because it disrupts the hydrogen bonding between guanines, therefore denaturing the G-quadruplex into single-stranded structures. Although we are not working with ssDNA, we aimed to compare $\mathrm{KCl}$ event properties with another alkali metal chloride that holds promise in the nanopore community. Therefore, we performed experiments using $10 \mathrm{mM} \mathrm{CsCl}$ inserted into $10 \mathrm{mM} \mathrm{CsCl}+\lambda$-DNA. The typical current trace and event signature is displayed in Figure 4d. 


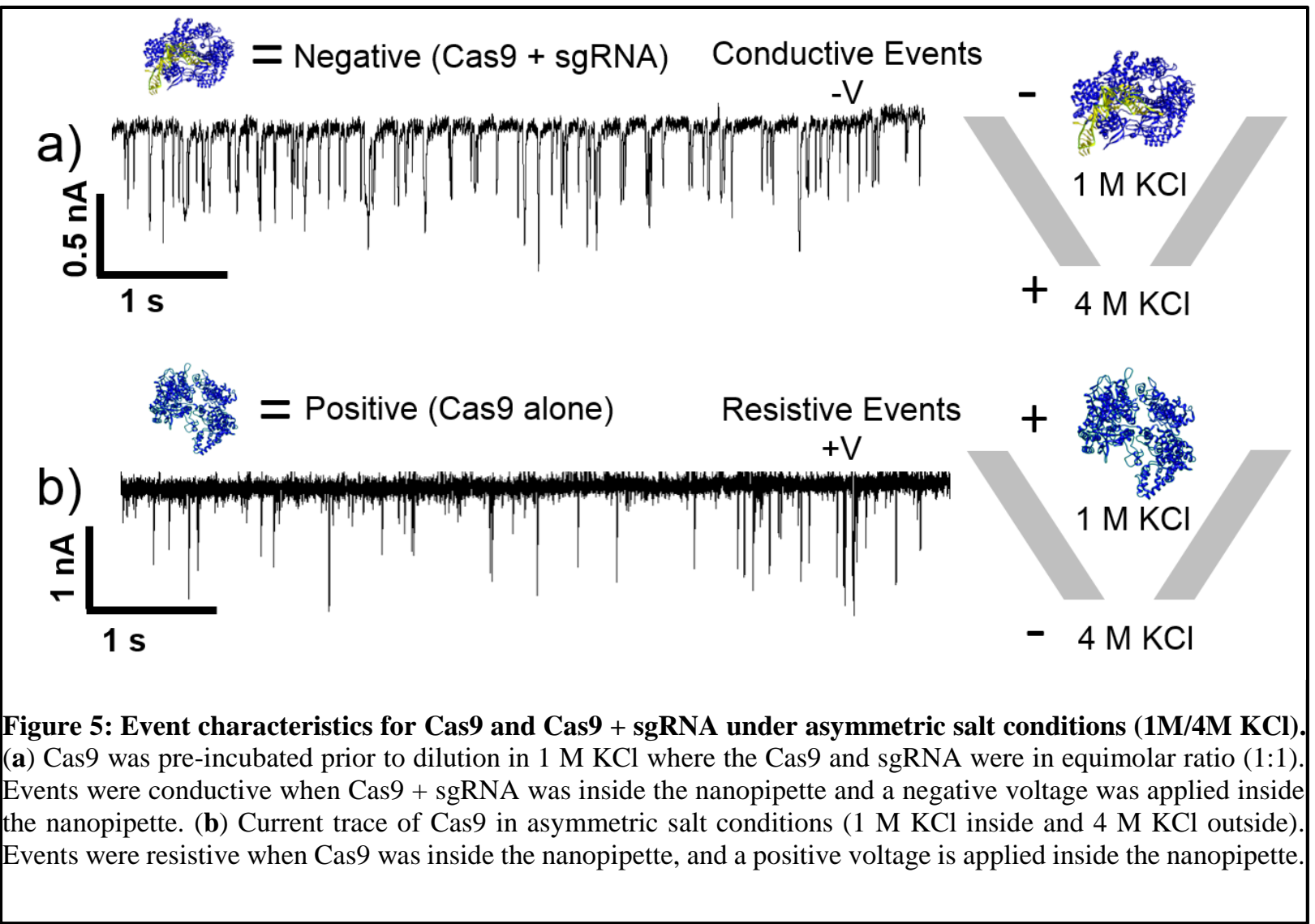

Similar to $\mathrm{KCl}$, we do not see a voltage dependence on event shape with $\mathrm{CsCl}$, which is not surprising considering that $\mathrm{K}^{+}$and $\mathrm{Cs}^{+}$have nearly the same diffusion coefficient ${ }^{42}$. For confirmation, a pore with a conductance of $1.47 \mathrm{nS}$ ( $14 \pm 2 \mathrm{~nm}$ diameter) was used with $\lambda$-DNA. Under low ionic strength conditions, we applied voltages of $-300 \mathrm{mV},-400 \mathrm{mV},-500 \mathrm{mV}$, and $1000 \mathrm{mV}$ to witness any transition in event shape (Fig. 4e). All voltages resulted in CEs, which was predicted based on finite element analysis under the assumption that cation selective conditions yield CEs. Simulation results for $\mathrm{CsCl}$ can be found in Supplemental Figure 9, but were nearly identical due to the diffusion coefficients for $\mathrm{KCl}$ and $\mathrm{CsCl}$ being $2.02 \times 10^{-5}$ and $2.00 \times 10^{-}$ ${ }^{5} \mathrm{~cm}^{2} / \mathrm{s}$, respectively ${ }^{42}$.

According to the experimental and numerical evidence, flux imbalance seems to play a role in producing CEs. Using the asymmetric salt conditions, we showed that a flux imbalance can be generated that favors potassium ions. A reversed voltage polarity would therefore generate a flux imbalance that favors chloride ions. We chose to study the Cas9 protein because alone it carries a net positive charge (at $\mathrm{pH} 7.4$ ) and once bound to sgRNA, the complex becomes highly negatively charged $^{43}$. The $\mathrm{pH}$ was not changed since this may have an unwanted effect on the nanopore's surface charge. The same asymmetric salt conditions were employed, as before, where $1 \mathrm{M} \mathrm{KCl}$ was inside the nanopipette and $4 \mathrm{M} \mathrm{KCl}$ was outside the nanopipette. The Cas9 protein was placed inside the nanopipette (in $1 \mathrm{M} \mathrm{KCl}$ ) with and without sgRNA (Fig. 5a and b, respectively). Similar to DNA, Cas9 + sgRNA produced CEs at negative voltages. Under this condition, potassium from 
the outside $(4 \mathrm{M} \mathrm{KCl})$ is driven into the pipette. After pre-incubating Cas9 with sgRNA (equimolar for 1 hour at room temperature), the complex was diluted in $1 \mathrm{M} \mathrm{KCl}$ and placed inside the nanopipette. At positive voltages, Cas9 produced REs. At this condition, chloride from the outside $(4 \mathrm{M} \mathrm{KCl})$ is driven into the pipette. The events at positive voltage could indeed be from either Cas9 + sgRNA or sgRNA alone since both are negatively charged. However Cas9 binding of sgRNA is typically fast with slow reversible reaction kinetics ${ }^{44}$.

How a flux imbalance yields CEs has yet to be addressed. The working hypothesis currently is that stored charges can accumulate at the nanopipette tip effectively acting as a capacitor in series with the highly resistive nanopore. Since the voltage at the extreme ends of the fluidic reservoirs are clamped, charge build-up (i.e. potassium) tends to generate a voltage that, in turn, lowers the effective voltage at the pore. Importantly, this effect does not occur with chloride since a negatively charged ion will avoid the negatively charged surfaces within the nanopipettes taper. We speculate that a DNA-occupied pore transiently stops EOF pumping and thereby lowers the stored charge inside the nanopore and that the capacitor discharges current proportional to the blocked EOF. Finite element methods demonstrate the accumulation of charge inside the glass pore (Fig. 6a). The increase in stored charge with applied voltage is a characteristic trait of an ionic capacitor. Upon solving for the effective capacitance, we obtain a value of $4 \times 10^{-17}$ Farads, as described in Figure $6 \mathrm{~b}$. The timescale of charging and discharging accumulated charge is also fast (3-5 $\mu$ s to reach steady state space charge density; Fig. 6c). The occupied pore will always have a higher resistance to ion flux as shown in Figure 6d. In this scenario, the only way to increase current is to change the voltage to a higher absolute value. We believe that charge storage and dissipation impacts the voltage in a dynamic way throughout the translocation process. A circuit model (Fig. 6d inset) is proposed where in translocation events turns a switch that blocks the charging of the nanopore capacitor. For a charged capacitor (i.e. open pore), the applied voltage will be distributed over the capacitor and the resistive nanopore. As a translocation event occurs, not only is the full applied voltage transferred to the resistive nanopore, but stored charge may also contribute to the overall current measured by the electrodes.

\section{Outlook}

Ionic-generated potentials are typically named according to the principle in which they are generated. For example, diffusion potentials, streaming potentials, and exclusion potentials ${ }^{45}$. Nevertheless, charge separation is a commonality of these potentials as well as our capacitor model which ultimately could generate voltage and current transients. Data thus far supports the hypothesis that a flux imbalance plays an important role in the generation of CEs. The existence of CEs with PEG (e.g. using a $0.43 \mathrm{nS}$ pore) further demonstrated that charged analytes are not a pre-requisite for CEs, but may indeed have an important role depending on the pore size. For example, a $2.63 \mathrm{nS}$ pore filled with $10 \mathrm{mM} \mathrm{KCl}$ produced CEs when DNA was the analyte, and REs for PEG at the same conditions. We speculate that the analyte and its concentration in the reservoir can transiently impact a pore's flux imbalance via translocation, or indirectly via interactions with the glass surface (i.e. outside the pore). For example, adsorbed molecules on the glass surface will hinder EOF pumping velocities and therefore the flux imbalance. Nevertheless, the evidence here demonstrates the importance of the pore's charged surface, voltage-bias, and associated electro-hydrodynamics in generating CEs. 
a)

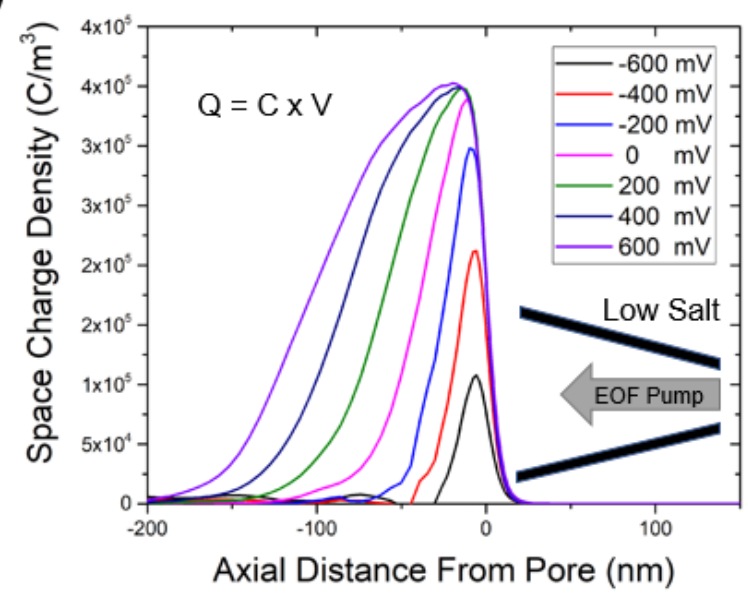

c)

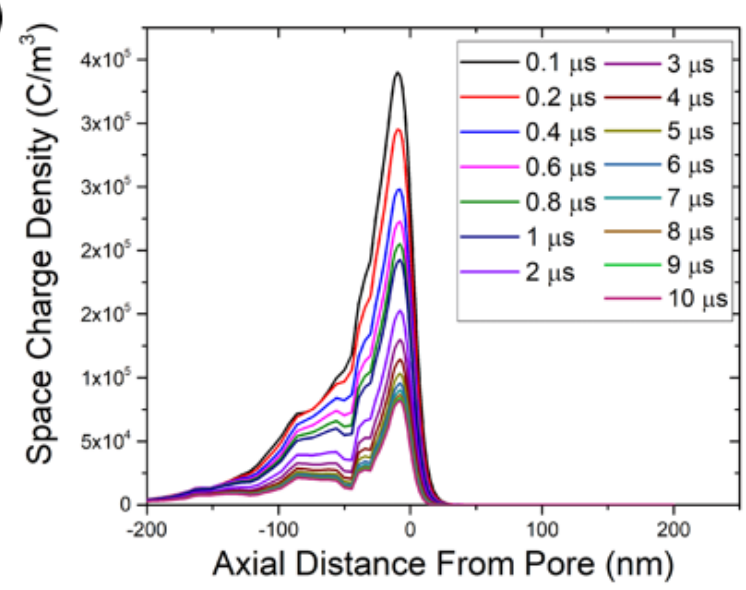

b)

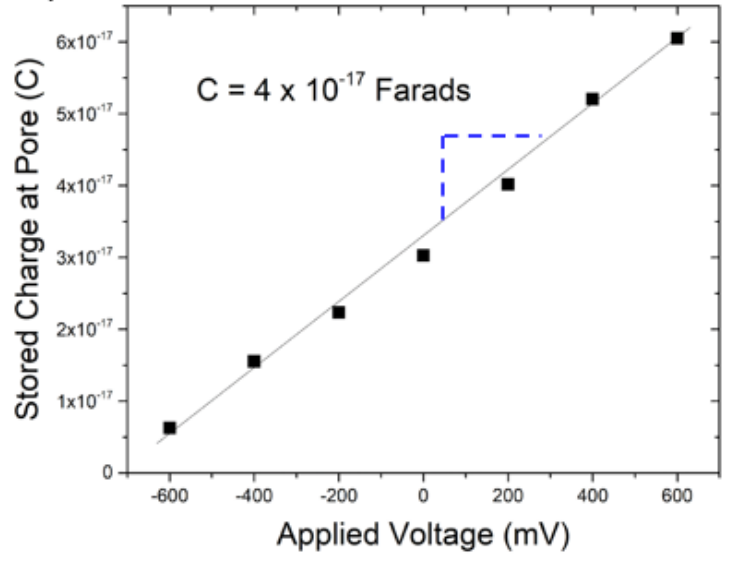

d)

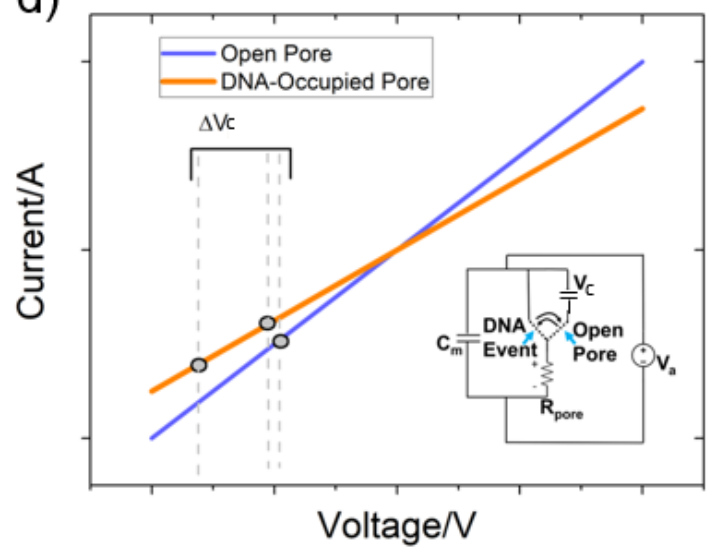

Figure 6: Stored charge model for flux imbalances. (a) Space charge density within a glass nanopore; axial distance of zero corresponds to the tip of the nanopipette. Pore diameter was $20 \mathrm{~nm}$ and was modelled with $10 \mathrm{mM} \mathrm{KCl}$. (b) Integrated stored charge within the nanopore as a function of applied voltage; capacitance is equal to the slope $\left(4 \times 10^{-17}\right.$ F). (c) Timescale of charge dispersion once EOF pumping is removed (voltage bias $=-600 \mathrm{mV}$ ). $(\mathbf{d})$ Proposed mechanism of how a nanopipette with potassium ions stored within the taper can yield CEs. An occupied pore will always act to reduce the current-voltage characteristics of the pore however the translocation event itself can cause transient blockage of potassium ions which act to charge the nanopore capacitor. The net result is that the pore experiences a voltage transient that shifts the voltage to a higher absolute value. Small voltages may yield resistive events (such as $\lambda$-DNA in $\mathrm{LiCl})$ but will transition to conductive events at higher voltages $(\lambda$-DNA in $\mathrm{LiCl})$.

In this study, we described multiple electro-hydrodynamic effects that influence EOFdriven DNA translocations under low ionic strength conditions. We have found that EOF can be used in various alkali chlorides and be used to translocate (un)charged molecules. Confirmation that EOF capture volume resides along the sides of the tip aperture and directs flow inward has been shown. The resulting current enhancement or reduction dependence on pore size can be explained by a pore's flux imbalance. Secondly, we discovered a crossover point, independent of salt concentration and specific to $\mathrm{LiCl}$, by scanning the applied voltage from $-300 \mathrm{mV}$ to $-900 \mathrm{mV}$. We show that changing the electrolyte influences the event shape, SNR values, and event 
frequency. Finally, by utilizing salt gradients to generate a flux imbalance, extremely high signal to noise ratios were achieved. Such information is valuable in the pursuit of using solid-state nanopores to sequence polynucleotides and as a diagnostic test.

\section{Methods}

Nanopore fabrication began with quartz capillaries (Sutter Instrument Co.) of $7.5 \mathrm{~cm}$ in length, $1.00 \mathrm{~mm}$ in outer diameter, and $0.70 \mathrm{~mm}$ in inner diameter. Capillaries were plasma cleaned for five minutes prior to laser assisted machine pulling to remove any surface contaminations. After, quartz capillaries were placed within the P-2000 laser puller (Sutter Instrument Co.) machine where $\mathrm{a} \mathrm{CO}_{2}$ laser heated the center of the capillary while the ends were pulled away from each other. A one-line protocol was used: (1) HEAT: 630; FIL: 4; VEL: 61; DEL: 145; PUL: between 135 and 195. This resulted in two identical, conical nanopores. The heat duration was approximately $4.5 \mathrm{~s}$.

Electrodes were constructed using silver wire dipped in bleach for 30 minutes and then rinsed. Nanopores were then backfilled with either $10 \mathrm{mM} \mathrm{KCl}, \mathrm{LiCl}$, or CsCl. An optical microscope was used to inspect the nanopores at this stage for any irregularities. Once the nanopore had been inspected, it was secured in our Axopatch set-up. Electrodes were then placed inside the pore and the solution containing $\lambda$-DNA. The Axopatch 200B patch-clamp amplifier (Molecular Devices, USA) was used in voltage clamp mode to measure the ionic current changes. The gain was optimized prior to each experiment and the signal was filtered with a low-pass filter at $5 \mathrm{kHz}$. Data analysis for DNA translocations and folding were performed using a custom MATLAB code.

For the microscopy experiments, $\lambda$-DNA with a stock concentration of $500 \mu \mathrm{gml}^{-1}$ was purchased from New England Biolabs. Dilutions were performed in either $10 \mathrm{mM} \mathrm{KCl}$ to create a $500 \mathrm{pM}$ concentration of $\lambda$-DNA. Afterwards, $\lambda$-DNA was incubated with YOYO-1 (Molecular Probes) for 30 minutes. Videos and images were captured using a $60 \mathrm{X}$ water-immersion objective.

COMSOL Multiphysics was used for modelling nanopipette geometries that were based on SEM and TEM images acquired from the same pipette pulling protocols that were used in sensing experiments. A 2D axisymmetric model was employed to reduce computational resources required. Once the geometries were created in COMSOL, the physics that were utilized included: laminar flow, transport of diluted species, and electrostatics. The electrostatics boundary condition for the glass was set at a surface charge density of $-1 \times 10^{-2} \mathrm{C} / \mathrm{m}^{2}$. In order to model electroosmotic flow, a volume force on the fluid was set to the space charge density of the ions in solution multiplied by the electric field vectors ( $\mathrm{r}$ and $\mathrm{z}$ vectors). Diffusion coefficients and mobility values were obtained from Lee et. al. ${ }^{42}$. All models were tested with different solvers, solving conditions, and reservoir sizes to ensure accuracy of results.

\section{Acknowledgements}

This work was partially supported by the Human Frontier Science Program (RGY0066/2018).

\section{Author Information}

Affiliations

Department of Bioengineering, University of California, Riverside, USA

Lauren S. Lastra, Nasim Farajpour, \& Kevin J. Freedman

Department of Biology, University of California, Riverside, USA 
Michelle Nguyen

\section{Contributions}

K.J.F. formulated the idea to explore low ionic strength solutions using DNA and nanopores. L.S.L. performed the experiments, devised analysis protocols, and carried out data analysis under guidance of K.J.F. M.N. performed preliminary experiments. N.F. simulated experiments using COMSOL. L.S.L and K.J.F. wrote the manuscript with contributions from all other authors.

\section{Corresponding Author}

Correspondence to Kevin J. Freedman (kfreedman@engr.ucr.edu)

\section{Ethics Declarations}

The authors declare no competing interests.

\section{References}

(1) Kasianowicz, J. J.; Brandin, E.; Branton, D.; Deamer, D. W. Characterization of Individual Polynucleotide Molecules Using a Membrane Channel. Proc. Natl. Acad. Sci. U.S.A. 1996, 93 (24), 13770-13773. https://doi.org/10.1073/pnas.93.24.13770.

(2) van Dorp, S.; Keyser, U. F.; Dekker, N. H.; Dekker, C.; Lemay, S. G. Origin of the Electrophoretic Force on DNA in Solid-State Nanopores. Nature Physics 2009, 5 (5), 347351. https://doi.org/10.1038/nphys1230.

(3) Kesselheim, S.; Müller, W.; Holm, C. Origin of Current Blockades in Nanopore Translocation Experiments. Phys. Rev. Lett. 2014, 112 (1), 018101. https://doi.org/10.1103/PhysRevLett.112.018101.

(4) Keyser, U. F.; Koeleman, B. N.; van Dorp, S.; Krapf, D.; Smeets, R. M. M.; Lemay, S. G.; Dekker, N. H.; Dekker, C. Direct Force Measurements on DNA in a Solid-State Nanopore. Nature Physics 2006, 2 (7), 473-477. https://doi.org/10.1038/nphys344.

(5) Haque, F.; Li, J.; Wu, H.-C.; Liang, X.-J.; Guo, P. Solid-State and Biological Nanopore for Real-Time Sensing of Single Chemical and Sequencing of DNA. Nano Today 2013, 8 (1), 56-74. https://doi.org/10.1016/j.nantod.2012.12.008.

(6) Dekker, C. Solid-State Nanopores. Nature Nanotechnology 2007, 2 (4), 209-215. https://doi.org/10.1038/nnano.2007.27.

(7) Chang, H.; Kosari, F.; Andreadakis, G.; Alam, M. A.; Vasmatzis, G.; Bashir, R. DNAMediated Fluctuations in Ionic Current through Silicon Oxide Nanopore Channels. Nano Lett 2004, 4 (8), 1551-1556. https://doi.org/10.1021/n1049267c.

(8) Smeets, R. M. M.; Keyser, U. F.; Krapf, D.; Wu, M.-Y.; Dekker, N. H.; Dekker, C. Salt Dependence of Ion Transport and DNA Translocation through Solid-State Nanopores. Nano Lett. 2006, 6 (1), 89-95. https://doi.org/10.1021/n1052107w.

(9) Wang, V.; Ermann, N.; Keyser, U. F. Current Enhancement in Solid-State Nanopores Depends on Three-Dimensional DNA Structure. Nano Lett. 2019, 19 (8), 5661-5666. https://doi.org/10.1021/acs.nanolett.9b02219.

(10) Steinbock, L. J.; Lucas, A.; Otto, O.; Keyser, U. F. Voltage-Driven Transport of Ions and DNA through Nanocapillaries. Electrophoresis 2012, 33 (23), 3480-3487. https://doi.org/10.1002/elps.201100663. 
(11) Ho, C.; Qiao, R.; Heng, J. B.; Chatterjee, A.; Timp, R. J.; Aluru, N. R.; Timp, G. Electrolytic Transport through a Synthetic Nanometer-Diameter Pore. Proc. Natl. Acad. Sci. U.S.A. 2005, 102 (30), 10445-10450. https://doi.org/10.1073/pnas.0500796102.

(12) Martins, D. C.; Chu, V.; Conde, J. P. The Effect of the Surface Functionalization and the Electrolyte Concentration on the Electrical Conductance of Silica Nanochannels.

Biomicrofluidics 2013, 7 (3), 034111. https://doi.org/10.1063/1.4811277.

(13) Stein, D.; Kruithof, M.; Dekker, C. Surface-Charge-Governed Ion Transport in Nanofluidic Channels. Phys. Rev. Lett. 2004, 93 (3), 035901. https://doi.org/10.1103/PhysRevLett.93.035901.

(14) Karnik, R.; Castelino, K.; Fan, R.; Yang, P.; Majumdar, A. Effects of Biological Reactions and Modifications on Conductance of Nanofluidic Channels. Nano Lett. 2005, 5 (9), 16381642. https://doi.org/10.1021/nl050966e.

(15) Siwy, Z.; Kosińska, I. D.; Fuliński, A.; Martin, C. R. Asymmetric Diffusion through Synthetic Nanopores. Phys. Rev. Lett. 2005, 94 (4), 048102. https://doi.org/10.1103/PhysRevLett.94.048102.

(16) Fan, R.; Karnik, R.; Yue, M.; Li, D.; Majumdar, A.; Yang, P. DNA Translocation in Inorganic Nanotubes. Nano Lett. 2005, 5 (9), 1633-1637. https://doi.org/10.1021/n10509677.

(17) Cui, S. Current Blockade in Nanopores in the Presence of Double-Stranded DNA and the Microscopic Mechanisms. J Phys Chem B 2010, 114 (5), 2015-2022. https://doi.org/10.1021/jp909564d.

(18) Cui, S. T. Counterion-Hopping along the Backbone of Single-Stranded DNA in Nanometer Pores: A Mechanism for Current Conduction. Phys. Rev. Lett. 2007, 98 (13), 138101. https://doi.org/10.1103/PhysRevLett.98.138101.

(19) Smeets, R. M. M.; Keyser, U. F.; Dekker, N. H.; Dekker, C. Noise in Solid-State Nanopores. Proceedings of the National Academy of Sciences 2008, 105 (2), 417-421. https://doi.org/10.1073/pnas.0705349105.

(20) Zhang, Y.; Wu, G.; Si, W.; Ma, J.; Yuan, Z.; Xie, X.; Liu, L.; Sha, J.; Li, D.; Chen, Y. Ionic Current Modulation from DNA Translocation through Nanopores under High Ionic Strength and Concentration Gradients. Nanoscale 2017, 9 (2), 930-939. https://doi.org/10.1039/C6NR08123A.

(21) Steinbock, L. J.; Bulushev, R. D.; Krishnan, S.; Raillon, C.; Radenovic, A. DNA Translocation through Low-Noise Glass Nanopores. ACS Nano 2013, 7 (12), 11255-11262. https://doi.org/10.1021/nn405029j.

(22) J. Steinbock, L.; Krishnan, S.; D. Bulushev, R.; Borgeaud, S.; Blokesch, M.; Feletti, L.; Radenovic, A. Probing the Size of Proteins with Glass Nanopores. Nanoscale 2014, 6 (23), 14380-14387. https://doi.org/10.1039/C4NR05001K.

(23) Ai, Y.; Zhang, M.; Joo, S. W.; Cheney, M. A.; Qian, S. Effects of Electroosmotic Flow on Ionic Current Rectification in Conical Nanopores. J. Phys. Chem. C 2010, 114 (9), 38833890. https://doi.org/10.1021/jp911773m.

(24) Steinbock, L. J.; Otto, O.; Chimerel, C.; Gornall, J.; Keyser, U. F. Detecting DNA Folding with Nanocapillaries. Nano Lett. 2010, 10 (7), 2493-2497. https://doi.org/10.1021/nl100997s.

(25) DeSorbo, W. Ultraviolet Effects and Aging Effects on Etching Characteristics of Fission Tracks in Polycarbonate Film. Nuclear Tracks 1979, 3 (1), 13-32. https://doi.org/10.1016/0191-278X(79)90026-X. 
(26) Sohi, A. N.; Beamish, E.; Tabard-Cossa, V.; Godin, M. DNA Capture by Nanopore Sensors under Flow. Anal. Chem. 2020, 92 (12), 8108-8116.

https://doi.org/10.1021/acs.analchem.9b05778.

(27) Charron, M.; Briggs, K.; King, S.; Waugh, M.; Tabard-Cossa, V. Precise DNA Concentration Measurements with Nanopores by Controlled Counting. Anal. Chem. 2019, 91 (19), 12228-12237. https://doi.org/10.1021/acs.analchem.9b01900.

(28) Wanunu, M.; Morrison, W.; Rabin, Y.; Grosberg, A. Y.; Meller, A. Electrostatic Focusing of Unlabelled DNA into Nanoscale Pores Using a Salt Gradient. Nature Nanotechnology 2010, 5 (2), 160-165. https://doi.org/10.1038/nnano.2009.379.

(29) Grosberg, A. Y.; Rabin, Y. DNA Capture into a Nanopore: Interplay of Diffusion and Electrohydrodynamics. J. Chem. Phys. 2010, 133 (16), 165102. https://doi.org/10.1063/1.3495481.

(30) Qiao, L.; Slater, G. W. Capture of Rod-like Molecules by a Nanopore: Defining an “Orientational Capture Radius.” J. Chem. Phys. 2020, 152 (14), 144902. https://doi.org/10.1063/5.0002044.

(31) Buyukdagli, S. Facilitated Polymer Capture by Charge Inverted Electroosmotic Flow in Voltage-Driven Polymer Translocation. Soft Matter 2018, 14 (18), 3541-3549. https://doi.org/10.1039/C8SM00620B.

(32) Laohakunakorn, N.; Thacker, V. V.; Muthukumar, M.; Keyser, U. F. Electroosmotic Flow Reversal Outside Glass Nanopores. Nano Lett. 2015, 15 (1), 695-702. https://doi.org/10.1021/nl504237k.

(33) Ermann, N.; Hanikel, N.; Wang, V.; Chen, K.; Weckman, N. E.; Keyser, U. F. Promoting Single-File DNA Translocations through Nanopores Using Electro-Osmotic Flow. J. Chem. Phys. 2018, 149 (16), 163311. https://doi.org/10.1063/1.5031010.

(34) Luan, B.; Aksimentiev, A. Electro-Osmotic Screening of the DNA Charge in a Nanopore. Phys. Rev. E 2008, 78 (2), 021912. https://doi.org/10.1103/PhysRevE.78.021912.

(35) Plesa, C.; Verschueren, D.; Pud, S.; van der Torre, J.; Ruitenberg, J. W.; Witteveen, M. J.; Jonsson, M. P.; Grosberg, A. Y.; Rabin, Y.; Dekker, C. Direct Observation of DNA Knots Using a Solid-State Nanopore. Nature Nanotech 2016, 11 (12), 1093-1097. https://doi.org/10.1038/nnano.2016.153.

(36) Kumar Sharma, R.; Agrawal, I.; Dai, L.; Doyle, P. S.; Garaj, S. Complex DNA Knots Detected with a Nanopore Sensor. Nature Communications 2019, 10 (1), 4473. https://doi.org/10.1038/s41467-019-12358-4.

(37) Li, J.; Gershow, M.; Stein, D.; Brandin, E.; Golovchenko, J. A. DNA Molecules and Configurations in a Solid-State Nanopore Microscope. Nature Materials 2003, 2 (9), 611615. https://doi.org/10.1038/nmat965.

(38) Storm, A. J.; Chen, J. H.; Zandbergen, H. W.; Dekker, C. Translocation of Double-Strand DNA through a Silicon Oxide Nanopore. Phys. Rev. E 2005, 71 (5), 051903. https://doi.org/10.1103/PhysRevE.71.051903.

(39) Colloid and Interface Chemistry for Water Quality Control; Elsevier, 2016. https://doi.org/10.1016/C2014-0-02506-3.

(40) Venta, K. E.; Zanjani, M. B.; Ye, X.; Danda, G.; Murray, C. B.; Lukes, J. R.; Drndić, M. Gold Nanorod Translocations and Charge Measurement through Solid-State Nanopores. Nano Lett. 2014, 14 (9), 5358-5364. https://doi.org/10.1021/nl502448s. 
(41) Kowalczyk, S. W.; Wells, D. B.; Aksimentiev, A.; Dekker, C. Slowing down DNA Translocation through a Nanopore in Lithium Chloride. Nano Lett. 2012, 12 (2), 10381044. https://doi.org/10.1021/nl204273h.

(42) Lee, S. H.; Rasaiah, J. C. Molecular Dynamics Simulation of Ion Mobility. 2. Alkali Metal and Halide Ions Using the SPC/E Model for Water at $25^{\circ}$ C. J. Phys. Chem. 1996, 100 (4), 1420-1425. https://doi.org/10.1021/jp953050c.

(43) Wang, P.; Zhang, L.; Xie, Y.; Wang, N.; Tang, R.; Zheng, W.; Jiang, X. Genome Editing for Cancer Therapy: Delivery of Cas9 Protein/SgRNA Plasmid via a Gold Nanocluster/Lipid Core-Shell Nanocarrier. Adv Sci (Weinh) 2017, 4 (11). https://doi.org/10.1002/advs.201700175.

(44) Raper, A. T.; Stephenson, A. A.; Suo, Z. Functional Insights Revealed by the Kinetic Mechanism of CRISPR/Cas9. J. Am. Chem. Soc. 2018, 140 (8), 2971-2984. https://doi.org/10.1021/jacs.7b13047.

(45) He, Y.; Tsutsui, M.; Scheicher, R. H.; Miao, X. S.; Taniguchi, M. Salt-Gradient Approach for Regulating Capture-to-Translocation Dynamics of DNA with Nanochannel Sensors. ACS Sens. 2016, 1 (6), 807-816. https://doi.org/10.1021/acssensors.6b00176. 